\title{
Gastropexy Using MIC-KEY Tube in a Dog with Esophageal-Gastric Leiomyoma
}

\section{Yoon Hee Kim² \\ Kyong-in Shin \\ Yeon-Jung Hong ${ }^{1}$ \\ Ulsoo $\mathrm{Choi}^{2, *}$}

${ }^{1}$ Western Animal Medical Center, Seoul 04101, Korea

${ }^{2}$ College of Veterinary Medicine, Jeonbuk National University, Iksan 54596, Korea
*Correspondence: uschoi@jbnu.ac.kr

\section{ORCID}

Yoon Hee Kim:

https://orcid.org/0000-0002-0651-8922

Ulsoo Choi:

https://orcid.org/0000-0002-3310-5533

Copyright $\odot$ The Korean Society of Veterinary Clinics
Abstract An 8-year-old neutralized male Dachshund was presented with severe vomiting, anorexia, and weight loss for two weeks. The patient had to feed in a standing position due to problem of vomiting immediately after meal. Serum chemistry and CBC results were all within reference limits. CT imaging revealed a mass distributed widely in the caudal part of the esophagus including the sphincter and the gastric cardia. Complete surgical removal was difficult because of adhesion to the caudal vena cava. A partial surgical excision and gastropexy with MIC-key feeding tube were performed under the owner's permission. Histologically, the mass was interpreted as leiomyoma. This patient is doing well at present time, six years after the tube installation in 2015.

Key words leiomyoma, gastropexy, mega-esophagus, MIC-tube, dog. 


\section{Introduction}

Esophageal tumor is known to account for less than < $0.5 \%$ of all neoplasia cases in dogs and cats. It occurs rarely as in humans (8). Among these cases, benign esophageal tumors including leiomyoma $(2,9)$ and plasmacytoma (4) are mainly located in the caudal part of the esophagus including the lower esophageal sphincter. On the other hand, malignant tumors include squamous cell carcinoma, leiomyosarcoma, firbrosarcoma, oseteosarcoma, and sarcoma caused by Spirocera lupi infection.

Clinical signs associated with tumors might be asymptomatic or vary. When tumors cause obstruction of the esophagus and a megaesophagus, it may appear progressive dysphagia, regurgitation, difficulty swallowing, vomiting, dehydration, and weight loss (10). Esophageal tumors can be diagnosed using plain radiography, contrast esophagram, computed tomography (CT) imaging, and endoscopy (11). For a definitive diagnosis, surgical biopsy and histopathology are needed. In dogs with esophageal tumor, possible treatments include local tumor debulking, surgical excision, radiation therapy, chemotherapy, neoplastic stricture dilation, palliative esophageal stents, and photodynamic therapy $(2,3,12)$.

Rolfe et al. (9) have reported three dogs with benign esophageal leiomyoma successfully treated by surgical excision. However, if the patient has a tumor adjacent to the esophageal sphincter, it is difficult to achieve complete resection. If there is a complication such as megaesophagus, it is difficult to improve the quality of life with a simple operation. This is the first case of gastropexy using MIC-KEY feeding tube in a dog with leiomyoma that is difficult to remove surgically.

\section{Case Presentation}

An 8-year-old neutered female dachshund was referred to the Western Animal Medical Center (AMC) for severe vomiting and loss of appetite that lasted more than two weeks. Exploratory laparotomy was performed one year ago to determine the cause before the patient was referred to the Western AMC and any remarkable finding was not found. Chronic intermittent vomiting persisted for more than one year. The owner was recommended to feed the dog in a standing position due to problem of vomiting immediately after meal.

At the time of referral admission, the dog was slightly lean with a body weight of $3.5 \mathrm{~kg}$. The dog showed moderate dehydration based on dry mucous membrane and reduced
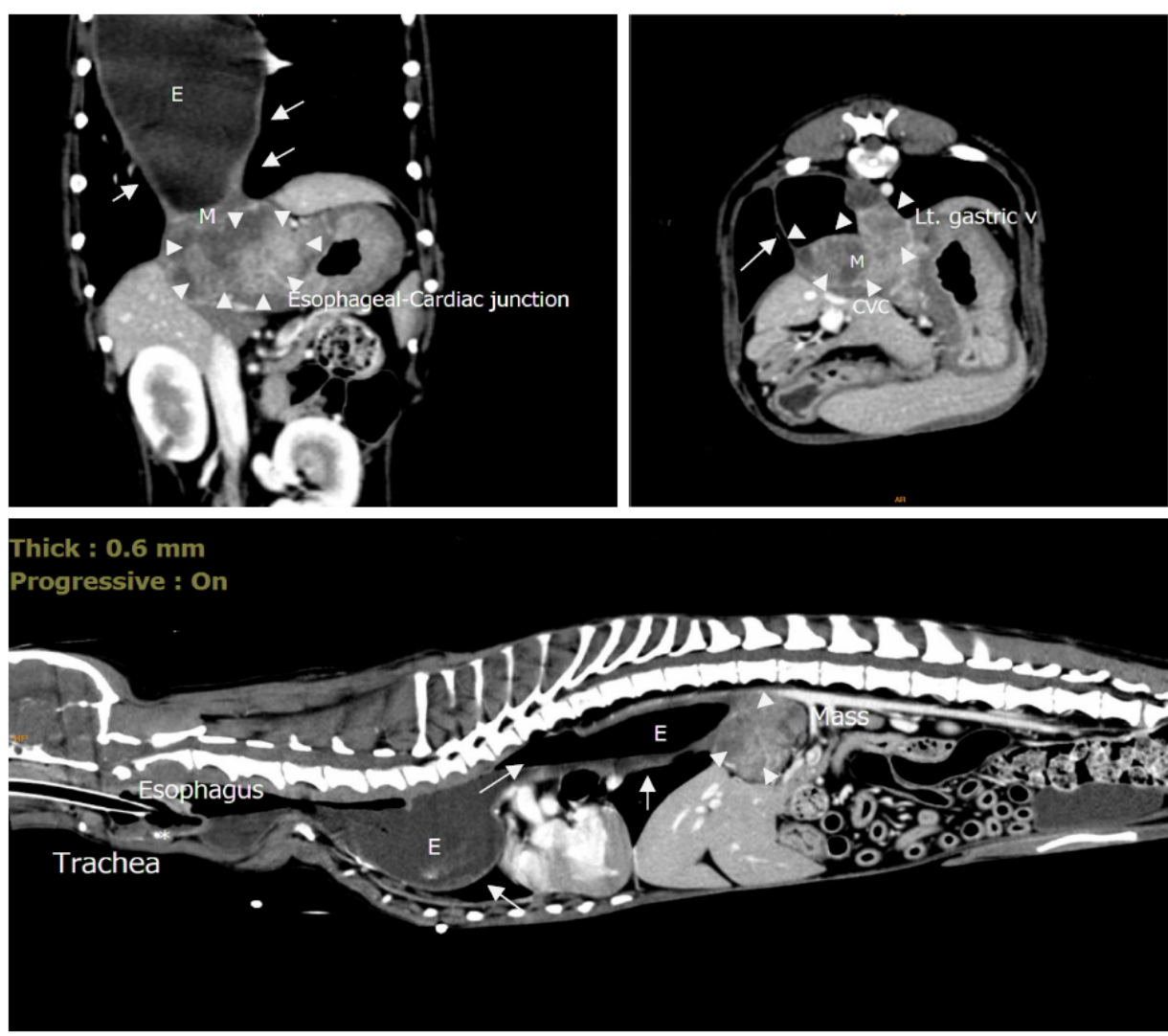

Fig. 1. Dorsal, transverse, and sagittal CT images of the megaesophagus, esophageal, and gastric mass. A mass showing a heterogeneous contrast enhancement was found from the distal esophagus to above the gastric cardia. The left gastric vein was judged to be the feeding vessel of the mass. The possibility of adhesion between the left/right gastric vein and the posterior vena cava was confirmed. Esophagus (long arrow), E; mass, M (arrowhead). 
skin turgor. Serum chemistry and $C B C$ results were all within reference limits except low sodium level $(124 \mathrm{mEq} / \mathrm{L}$, reference interval: $144-160 \mathrm{mEq} / \mathrm{L})$ and chloride level $(80 \mathrm{mEq} / \mathrm{L}$; reference interval: $105-119 \mathrm{mEq} / \mathrm{L}$ ) due to vomiting and dehydration. On thoracic radiography, an ovoid soft tissue mass was observed in the caudal esophagus region and a severe megaesophagus accompanied by gas was identified in the cranial part to the mass. Abdominal ultrasonography showed a slightly heterogeneous well-defined mass $(3 \times 2.5 \mathrm{~cm})$ at the level of the gastric cardia. CT scan was performed to confirm the origin of the mass, revealing severe esophageal enlargement (megaesophagus, as seen on the thoracic radiography) and fluid retention in the lumen (Fig. 1). Additionally, a $4.5 \times 5 \times 3 \mathrm{~cm}$ mass was found on CT scan, showing heterogeneous enhancement of the contrast from the distal esophagus to the caria of stomach. The left gastric vein was assumed to be the feeding vessel of the mass. Adhesion between the left/right gastric vein and the posterior vena cava was confirmed.

Because $C T$ imaging revealed that the mass was distributed widely in the caudal part of the esophagus including the sphincter and the cardia/body of the stomach, complete surgical removal was considered to be difficult due to suspicion of adhesion to the caudal vena cava. It was necessary to consider ways to reduce vomiting caused by megaesophagus and to provide adequate nutrition at the same time. At the decision of the owner, partial resection of the tumor and gastropexy with MIC-KEY Low-profile gastrostomy

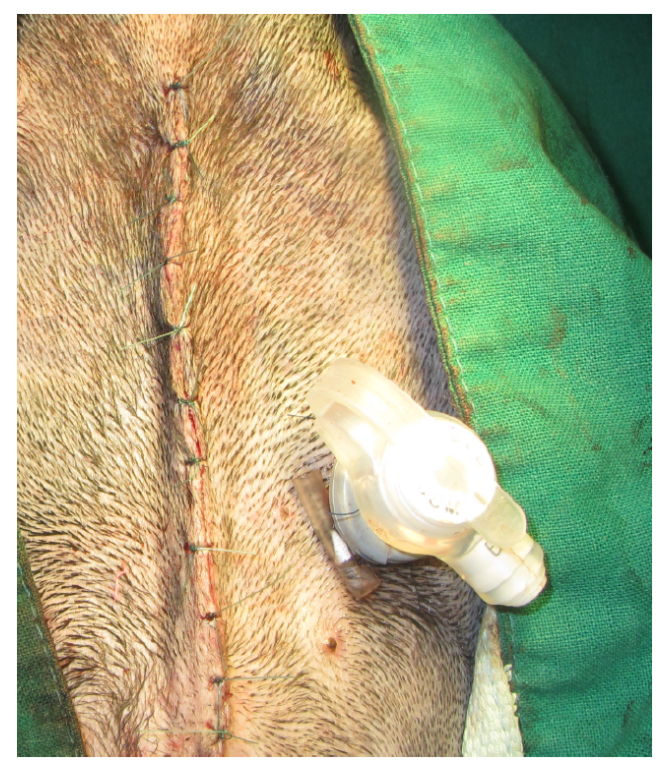

Fig. 2. Fixed MIC-tube through the abdominal wall via gastropexy. Under general anesthesia, partial resection of the gastric mass was performed and a MIC-KEY tube was attached. feeding tube (Kimberly-Clark, 12 Fr, $0.8 \mathrm{~cm}$ ) were performed under general anesthesia (Fig. 2). The excised tissue was fixed in 10\% neutral buffered formalin and submitted for histopathological examination to IDEXX Laboratories Korea. Stromal neoplasm consists of interlacing bundles of plump spindle-shaped cells in a very light fibrovascular stroma (Fig. 3). Neoplastic cells have abundant homogenous eosinophilic cytoplasm with central cigar-shaped uniform nuclei and negligible mitotic activity. Since only incisional sections were submitted, accurate assessment of margins could not be done. These characteristic histopathologic features were consistent with a leiomyoma.

The patient was fed sequentially $\mathrm{a} / \mathrm{d}$ and grinded dry feed through the tube. Its body weight increased close to normal (BCS 3/5, $4.5 \mathrm{~kg}$ ) without vomiting during re-visits every three months after discharge. Even at the third recheck (11 months after surgery), thoracic radiograph showed no improvement in megaesophagus. This patient is doing well to the present time six years after the tube installation in 2015.

\section{Discussion}

Leiomyoma of smooth muscle origin is an uncommon tumor reported in canine esophagus and stomach $(7,8)$. This tumor is mainly an incidental finding during necropsy (2). It can cause clinical signs such as regurgitation, repeated vomiting, mild anemia, and weight loss. Most gastric leiomyomas occur in the cardia of the stomach or at the gastro-esophageal junction. The average age of affected dogs was 14.8 years (6). In another study, the incidence of gastric

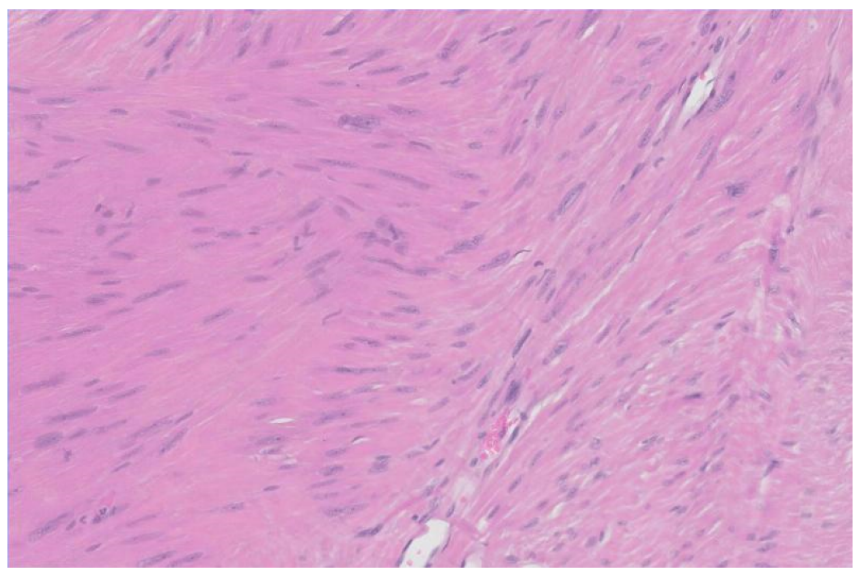

Fig. 3. Histology of partially excised mass. Stromal neoplasm consists of interlacing bundles of plump spindle-shaped cells in a very light fibrovascular stroma. Neoplastic cells have abundant homogenous eosinophilic cytoplasm with central cigar-shaped uniform nuclei and negligible mitotic activity. 
leiomyoma in laboratory Beagles tended to increase with age (2). The majority (80\%) of affected dogs were between 17 and 18 years of age (2). The gastric tumor of the present patient developed at a relatively young age of 8 years. It was accompanied by severe esophageal obstruction and resulting megaesophagus. According to Arnell et al. (1), all four dogs diagnosed as caudal esophageal neoplasia presented with megaesophagus in radiography. The dog in the present case also had intermittent vomiting for more than one year because of the megaesophagus. The cause of the megaesophagus was presumed to be due to narrowing of the caudal esophageal, weak esophageal sphincter tone, and dysmotility.

The diagnosis of an esophageal tumor is typically based on clinical signs, diagnostic imaging, and esophagoscopy. A barium esophagogram can detect the presence of either an intraluminal mass or obstructive lesion. CT is a useful method for measuring tumor size, determining metastasis, and planning of surgery. However, it has limitations in finding a tumor less than $1 \mathrm{~cm}$ in size. Endoscopy can visualize mucosal margins of esophagus and stomach tumor. It is a useful method for debulking and partial resection to relieve clinical signs. We did not perform barium contrast or endoscopy, making it difficult to determine the obstructive lesion of the mass before surgery.

In a report by Rolfe et al. (9), three dogs with esophageal leiomyoma in the area including the lower esophageal sphincter were managed successfully with surgery. Treatment includes surgical resection, radiation therapy, chemotherapy, neoplastic stricture dilation, palliative esophageal stent placement, and photodynamic therapy. Hansen et al. (5) has tried esophageal stent replacement in a patient diagnosed with esophageal squamous cell carcinoma. However, the patient's condition deteriorated. The patient was euthanized at 4 weeks after the procedure. In Korea, it is difficult to apply radiation and photodynamic therapy. When exploratory laparotomy was performed, neoplastic stricture dilation and palliative esophageal stent placement could be considered at first. We decided partial resection and attachment of MICkey feeding tube to alleviate clinical signs because the mass obstructed nearly all esophagus and gastric lumen. The low-profile gastrostomy feeding tube designed by Halyard Health is a low-adherent tube that can be nourished directly from the stomach in human patients (especially children). Similarly, it was selected because it well suited small dogs known to be difficult to manage due to their high activities. In addition, if the skin wound with the tube is fully recovered, the tube can be replaced without anesthesia. In this dog, a tube with a stroma length of $0.8 \mathrm{~cm}$ and an outer diameter of 16 Fr was used.

Conclusively, gastro-esophageal leiomyoma overlying the caudal esophagus including a lower sphincter and gastric cardia was confirmed by CT and histopathology. Partial excision of tumor and application of MIC-KEY feeding tube were performed. The patient has been doing well clinically without any side effects up to the present time, six years after the surgery. Thus, MIC-KEY feeding can be considered as an alternative for a patient with a gastroesophageal tumor that is growing slowly but difficult to remove completely.

\section{Conflicts of Interest}

The authors have no conflicting interests.

\section{References}

1. Arnell K, Hill S, Hart J, Richter K. Persistent regurgitation in four dogs with caudal esophageal neoplasia. J Am Anim Hosp Assoc 2013; 49: 58-63.

2. Culbertson R, Branam JE, Rosenblatt LS. Esophageal/gastric leiomyoma in the laboratory Beagle. J Am Vet Med Assoc 1983; 183: 1168-1171.

3. Farese JP, Bacon NJ, Ehrhart NP, Bush J, Ehrhart EJ, Withrow SJ. Oesophageal leiomyosarcoma in dogs: surgical management and clinical outcome of four cases. Vet Comp Oncol 2008; 6: 31-38.

4. Hamilton TA, Carpenter JL. Esophageal plasmacytoma in a dog. J Am Vet Med Assoc 1994; 204: 1210-1211.

5. Hansen K, Weisee C, Berent A. Palliative stenting for malignant esophageal obstruction in a dog. In: 6th Annual Meeting of the Veterinary Endoscopy Society. Cancun: Veterinary Endoscopy Society, 2009

6. Kerpsack SJ, Birchard SJ. Removal of leiomyomas and other noninvasive masses from the cardiac region of the canine stomach. J Am Anim Hosp Assoc 1994; 30: 500-504.

7. Patnaik AK, Hurvitz Al. Neoplasms of the digestive tract. In: Kirk RW, editor. Current veterinary therapy VI: small animal practice. Philadelphia: Saunders. 1977: 939-942

8. Ridgway RL, Suter PF. Clinical and radiographic signs in primary and metastatic esophageal neoplasms of the dog. J Am Vet Med Assoc 1979; 174: 700-704

9. Rolfe DS, Twedt DC, Seim HB. Chronic regurgitation or vomiting caused by esophageal leiomyoma in three dogs. J Am Anim Hosp Assoc 1996; 30: 425-430.

10. Theilen GH, Madewell BR. Tumors of the digestive tract. In: Theilen GH, Madewell BR, editors. Veterinary cancer medicine. 2nd ed. Philadelphia: Lea \& Febiger. 1987: 511-513.

11. Withrow SJ, Vail DM. Esophageal cancer. In: Withrow SJ, Vail DM, editors. Withrow \& MacEwen's small animal clinical oncology. 4th 
ed. St. Louis: Saunders Elsevier. 2007: 478-479.

12. Won JH, Lee JD, Wang HJ, Kim GE, Kim BW, Yim H, et al. Self-expandable covered metallic esophageal stent impregnat- ed with beta-emitting radionuclide: an experimental study in canine esophagus. Int J Radiat Oncol Biol Phys 2002; 53: 10051013. 